\title{
PCR fingerprinting of Trichophyton mentagrophytes var. interdigitale using polymorphic subrepeat loci in the rDNA nontranscribed spacer
}

\author{
Colin J. Jackson, ${ }^{1}$ Takashi Mochizuki ${ }^{2}$ and Richard C. Barton ${ }^{3}$ \\ ${ }^{1}$ Institute of Biological Sciences, University of Wales, Aberystwyth, Wales, UK \\ ${ }^{2}$ Department of Dermatology, Kanazawa Medical University, Uchinada, Japan \\ ${ }^{3}$ Mycology Reference Centre, Department of Microbiology, General Infirmary, and University of \\ Leeds, Leeds, UK
}

The sequence of the nontranscribed spacer (NTS) region of the rDNA of Trichophyton mentagrophytes var. interdigitale strain 2111 was determined, and three individual subrepeat loci identified. The first repeat region contained eight tandem copies of a degenerate 33-43 bp sequence, whilst the second had two complete and two partial $300 \mathrm{bp}$ repeats. The third locus contained six tandemly repetitive elements of between 67 and $89 \mathrm{bp}$, which showed sequence identity to the TrS2 repeats of Trichophyton rubrum. PCR amplification of the individual repetitive regions from 42 random isolates of $T$. mentagrophytes var. interdigitale identified fragment length polymorphisms at each locus. Sequence analysis of the PCR products revealed that the size variations resulted from differences in the copy number of each of the three sets of subrepeat elements, TmiSo, TmiS1 and TmiS2. In addition, some indels were present in the flanking regions of the TmiS1 repeats. Combining PCR fingerprints from each of the three polymorphic loci produced a total of 19 individual strain profiles. The method was rapid, reproducible and discriminatory, and the fragment patterns simple to interpret. PCR fingerprint analysis of variable tandem repeat loci

Received 20 April 2006 in the T. mentagrophytes var. interdigitale NTS represents a valuable molecular typing method for future epidemiological investigations in this species.

\section{INTRODUCTION}

Human-adapted dermatophyte species are the most prevalent fungal pathogens in the developed world. Although the clinical consequences of dermatophyte infections are generally benign, and serious sequelae from these infections are rare, medical costs associated with the treatment of dermatophytoses place a large burden on healthcare budgets. These have been conservatively estimated at $\$ 400$ million per annum in the USA alone (Drake et al., 1996; Smith et al., 1998). High rates of dermatophyte infection are maintained by widespread use of communal recreational facilities, such as gymnasiums, sports centres and public swimming baths. These environments afford optimal conditions for the transmission of tinea infections,

\footnotetext{
Abbreviations: LSU, large subunit; NTS, nontranscribed spacer; SRE, subrepeat element; SSU, small subunit; VIR, variable internal repeat.

The GenBank/EMBL/DDBJ accession number for the NTS region of $T$. mentagrophytes var. interdigitale, containing the coordinates of the three subrepeat loci, is DQ486866.

Supplementary data are available with the online version of this paper.
}

particularly 'athlete's foot' (tinea pedis). Once an infection is established, the wearing of sports shoes and other types of occlusive footwear provides an ideal microclimate in which these fungi can thrive, and may lead to nail infections (tinea unguium), which are inherently harder to treat. It has been estimated that there is a relapse rate of $10 \%$ a year in cases of tinea pedis treated with oral terbinafine (Takiuchi et al., 2005 ), while $22 \%$ of patients with tinea unguium treated with systemic antifungals relapse within 3 years (Tosti et al., 1998). The design of effective intervention measures to break the cycle of transmission is a central requirement for reducing the incidence of dermatophytoses. The epidemiological typing of fungal isolates is one means by which point sources of infection, routes of transmission, and sources of recurrence (i.e. reinfection with new strains or recrudescence of the original strain) can be accurately identified.

The two most common agents of tinea pedis are the anthropophilic dermatophytes Trichophyton mentagrophytes var. interdigitale and Trichophyton rubrum (Zaias \& Rebell, 2003). Both species cause very similar clinical conditions, but are quite well separated in the phylogeny 
Table 1. Details of $T$. mentagrophytes var. interdigitale isolates used in this study

Abbreviations: KMU, Kanazawa Medical University; NK, not known; ND, not done; NR, no result.

\begin{tabular}{|c|c|c|c|c|c|c|}
\hline \multirow[t]{2}{*}{ Isolate } & \multirow[t]{2}{*}{ Origin } & \multirow[t]{2}{*}{ Site } & \multicolumn{3}{|c|}{ Strain PCR type } & \multirow[t]{2}{*}{ RFLP $^{\star}$ group } \\
\hline & & & So & S1 & S2 & \\
\hline Av.A27960† & Belgium & NK & B & 0 & I & ND \\
\hline EK1471 & UK & Chest & A & 1 & I & ND \\
\hline EP0843 & UK & NK & $\mathrm{C}$ & 2 & I & P-6 \\
\hline EP1127 & UK & NK & $\mathrm{I}$ & 7 & IA & $\mathrm{P}-3$ \\
\hline 2882 & UK & NK & G & 1 & II & $\mathrm{P}-1$ \\
\hline 0388 & UK & NK & $\mathrm{F}$ & 1 & II & ND \\
\hline 1553 & UK & NK & $\mathrm{C}$ & 2 & II & ND \\
\hline T2.261 & UK & NK & $\mathrm{C}$ & 2 & II & P-6 \\
\hline 2111 & UK & NK & $\mathrm{C}$ & 2 & II & $\mathrm{P}-1$ \\
\hline Tmi.dysg & UK & NK & $\mathrm{D}$ & 2 & II & ND \\
\hline 0269 & UK & NK & $\mathrm{D}$ & 2 & II & ND \\
\hline $\mathrm{T} 2.2450$ & NK & NK & $\mathrm{D}$ & 2 & II & ND \\
\hline 2768 & UK & NK & G & 2 & II & ND \\
\hline 7435 & UK & NK & $\mathrm{D}$ & 2 & II & ND \\
\hline 3964 & UK & NK & $\mathrm{D}$ & 2 & II & ND \\
\hline SM8796 & Japan & Foot & $\mathrm{D}$ & 2 & II & P-1 \\
\hline KMU.Kdy & Japan & NK & $\mathrm{D}$ & 2 & II & P-1 \\
\hline KMU.Tnk & Japan & NK & $\mathrm{D}$ & 2 & II & P-1 \\
\hline KMU.Ysh & Japan & Foot & $\mathrm{D}$ & 2 & II & $\mathrm{P}-1$ \\
\hline 2710 & UK & NK & $\mathrm{D}$ & 2 & II & P-1 \\
\hline EP1039 & UK & Toe & $\mathrm{D}$ & 2 & II & P-1 \\
\hline EP1119 & UK & Toe web & $\mathrm{D}$ & 2 & II & P-1 \\
\hline EP0904 & UK & NK & $\mathrm{D}$ & 2 & II & ND \\
\hline EP0939 & UK & Foot & $\mathrm{D}$ & 2 & II & P-1 \\
\hline EP9294 & UK & NK & $\mathrm{D}$ & 2 & II & ND \\
\hline 6679 & UK & NK & $\mathrm{D}$ & 2 & II & P-1 \\
\hline KMU.Tkd & Japan & NK & $\mathrm{F}$ & 2 & II & $\mathrm{P}-1$ \\
\hline EP0914 & UK & Toenail & F & 2 & II & P-3 \\
\hline 2035 & UK & NK & $\mathrm{D}$ & 3 & II & P-9 \\
\hline KMU.Aik & Japan & Palm & $\mathrm{D}$ & 4 & II & P-5 \\
\hline 4605 & UK & NK & $\mathrm{D}$ & 6 & II & $\mathrm{P}-2$ \\
\hline EK4354 & UK & Chin & $\mathrm{J}$ & 5 & II & P-11 \\
\hline EP1159 & UK & Nail & $\mathrm{D}$ & 6 & II & P-4 \\
\hline 3083 & UK & NK & $\mathrm{D}$ & 6 & II & $\mathrm{P}-4$ \\
\hline T2.36 & NK & NK & $\mathrm{D}$ & 7 & II & ND \\
\hline 4153 & UK & NK & $\mathrm{D}$ & 7 & II & P-5 \\
\hline EG5704 & UK & Shin & $\mathrm{D}$ & 7 & II & P12 \\
\hline EP1174 & UK & Toenail & $\mathrm{D}$ & 7 & IV & P-2 \\
\hline EF2608 & UK & Palm & $\mathrm{E}$ & 8 & III & P-10 \\
\hline 3850 & UK & NK & $\mathrm{D}$ & 9 & II & $\mathrm{P}-4$ \\
\hline 8070 & UK & NK & $\mathrm{H}$ & 10 & II & P-7 \\
\hline SM8774 & India & NK & NR & NR & II & P-1 \\
\hline SM8779 & India & NK & $\mathrm{NR}$ & NR & II & P-1 \\
\hline
\end{tabular}

*The RFLP type as determined using a probe targeted to the NTS region (Mochizuki et al., 2003).

$\dagger$ Av.A27960, A. vanbreuseghemii isolate from Professor R. Vanbreuseghem, Antwerpen, Belgium.

¥The original designations for the two isolates from the Indian subcontinent were Menon 187 for SM8774 and Menon 302 for SM8779. of the genus. T. mentagrophytes var. interdigitale has been placed in a separate species, $T$. interdigitale, by some authors (Gräser et al., 1999a), but this group of dermatophytes, isolated almost exclusively from the skin and nails of human feet, is distinctive regardless of name. Isolates of $T$. mentagrophytes var. interdigitale can show substantial variation in colony morphology and appearance, but these characters can alter greatly on subculture, and are therefore inappropriate for use as phenotypic strain markers.

Molecular subtyping offers an alternative way of identifying individual fungal isolates for epidemiological purposes. We have previously developed a genotyping procedure based upon variable tandem subrepeat elements (SREs) in the rDNA gene cluster of T. rubrum (Jackson et al., 2000). Recently, T. mentagrophytes var. interdigitale has also been shown to possess genetic polymorphisms that map to the rDNA (Mochizuki et al., 2003). Here, we report the molecular characterization of the nontranscribed spacer (NTS) of the rDNA of T. mentagrophytes var. interdigitale, and identify three variable loci within it that consist of tandemly repetitive subelements. PCR amplification of each of these polymorphic NTS loci generated simple, discriminatory patterns, which when combined produced a robust molecular subtyping scheme for this dermatophyte.

\section{METHODS}

Fungal isolates. The strains used and their origins are listed in Table 1. All were randomly selected isolates cultured from clinical samples submitted from patients with dermatophytoses, though the exact specimen type was not available in some cases. Twenty samples were obtained from the Mycology Reference Centre at Leeds University Medical School, Leeds, UK, and 14 from the Department of Dermatology at St Thomas's Hospital, London. Strains from Japan (six), India (two) and Belgium (one) were provided by the Department of Dermatology, Kanazawa Medical University, Japan. The clinical isolates were identified on the basis of microscopy and colony morphology from the primary cultures, and by restriction profiling of ITS regions 1 and 2 (Jackson et al., 1999; Mochizuki et al., 2003).

DNA isolation. Nucleic acids were extracted from fungal cultures as described previously (Jackson et al., 1999), with the following modifications. The lysis buffer contained $100 \mu \mathrm{g}$ proteinase $\mathrm{K} \mathrm{ml}^{-1}$, and the initial extraction of the lysate with ice-cold chloroform was omitted.

PCR amplification and cloning of the NTS region. The NTS region was successfully amplified from three $T$. mentagrophytes var. interdigitale isolates (2111, EP0914 and KMU.Aik) using a modification of the procedure described previously (Jackson et al., 2000). The PCR reaction contained 1× Expand Long Buffer 3, $2.75 \mathrm{mM}$ magnesium chloride, $500 \mu \mathrm{M}$ each deoxynucleoside triphosphate (dATP, dCTP, dTTP, dGTP), $300 \mathrm{nM}$ of each conserved primer [forward 25SCON2, reverse NS1-R (White et al., 1990; Table 2)], 3.75 units of Expand Long high fidelity Taq DNA polymerase (Expand Long Template PCR System, Roche) and $1 \mu$ genomic DNA template $(\sim 20 \mathrm{ng})$, in a total volume of $50 \mu \mathrm{l}$. The thermal profile was altered to optimize the yield, with an initial denaturation of $95^{\circ} \mathrm{C}$ for $2.5 \mathrm{~min}$, followed by ten cycles of denaturation at $95^{\circ} \mathrm{C}$ for $20 \mathrm{~s}$, annealing at $55^{\circ} \mathrm{C}$ for $35 \mathrm{~s}$ and extension at $68^{\circ} \mathrm{C}$ for $75 \mathrm{~s}$. 
Table 2. PCR primers used for amplification of the NTS region, and for the three fingerprinting assays.

\begin{tabular}{|c|c|}
\hline Primer & Sequence \\
\hline \multicolumn{2}{|c|}{ Primers for amplification of the Tmi NTS region } \\
\hline Forward primer $25 \mathrm{SCON} 2$ & $5^{\prime}$ TAG ACC GTC GTG AGA CAG \\
\hline Reverse primer NS1-R & 5' GAG ACA AGC ATA TGA CTA C \\
\hline \multicolumn{2}{|c|}{ Primers for amplification of the S0 tandem repeat region } \\
\hline Forward primer TmiSOF & 5' CGA AGG ATA CTG TGG AAG ATG \\
\hline Reverse primer TmiSOR & 5' GCA ACA TAA GTG TAC AGC TG \\
\hline \multicolumn{2}{|c|}{ Primers for amplification of the $S 1$ tandem repeat region } \\
\hline Forward primer TmiS1F & $5^{\prime}$ CAG CTG TAC ACT TAT GTT GC \\
\hline Reverse primer TmiS1R & $5^{\prime}$ TCG TTC GCC TCG AAG ACG CAC \\
\hline \multicolumn{2}{|c|}{ Primers for amplification of the $S 2$ tandem repeat region } \\
\hline Forward primer TmiS2F & 5' GAC CTT CAT TCT AGC TAT G \\
\hline Reverse primer TmiS2R & 5' CCT ATC GTA CGA GAA CGT TAG \\
\hline
\end{tabular}

This was followed by 20 cycles using exactly the same thermal profile, but with an additional $20 \mathrm{~s}$ of extension time on each repeated cycle. A terminal extension of $4 \mathrm{~min}$ at $68^{\circ} \mathrm{C}$ completed the reaction.

The NTS PCR product was purified by gel electrophoresis, and subcloned into the vector pGEM-T Easy (Promega). The NTS from strain 2111 was selected for sequencing based on the low-complexity rDNA pattern (type P-1) shown by this isolate in a previous study (Mochizuki et al., 2003). The NTS was sequenced on the sense and antisense strands using a primer-walking strategy. Reactions were carried out using dye terminator chemistry on an Applied Biosystems 377 automated sequencer. Repetitive tracts of DNA were initially located using a nucleic acid dot-matrix self-comparison program (http:// arbl.cvmbs.colostate.edu/molkit/dnadot/), and verified manually. Secondary structure predictions for sequence at the $3^{\prime}$ end of the large subunit (LSU) rRNA gene were carried out using the RNAFold program (http://rna.tbi.univie.ac.at/cgi-bin/RNAfold.cgi).

PCR amplification of the subrepeat regions S0, S1 and S2. A single generic PCR reaction mixture and thermal profile was developed to allow amplification of all three polymorphic loci using the same conditions. PCR primers were designed for the specific amplification of each of the three loci containing repeats TmiS0, TmiS1 and TmiS2 (Table 2). The PCR mixture contained $1 \times$ reaction buffer (Promega), $1.5 \mathrm{mM}$ magnesium chloride, $20 \mathrm{pmol}$ each primer, $0.2 \mathrm{mM}$ each dNTP and 1.25 units Taq polymerase (Promega), in a total volume of $49 \mu \mathrm{l}$. One microlitre of genomic DNA template was added per reaction containing $>10^{4}$ copies of the target sequence ( $\sim 20 \mathrm{ng}$ per reaction). Thermal cycling conditions were an initial denaturation at $95^{\circ} \mathrm{C}$ for $3 \mathrm{~min}$, followed by 30 cycles of denaturation at $95^{\circ} \mathrm{C}$ for $30 \mathrm{~s}$, annealing at $55^{\circ} \mathrm{C}$ for $30 \mathrm{~s}$ and extension at $72{ }^{\circ} \mathrm{C}$ for $90 \mathrm{~s}$. A terminal extension of $5 \mathrm{~min}$ at $72^{\circ} \mathrm{C}$ completed the reaction. Polymorphisms between PCR products from each locus were identified by comparative gel electrophoresis

\section{RESULTS AND DISCUSSION}

\section{Characterization of the $T$. mentagrophytes var. interdigitale NTS region}

The NTS region was 3322 bp in length, and bounded upstream by the $3^{\prime}$ terminus of the LSU rDNA gene, and downstream by the $5^{\prime}$ end of the small subunit (SSU) rDNA gene. The end of the LSU rDNA gene was predicted by comparison with sequence from the experimentally determined $3^{\prime}$ terminus of the Candida albicans $25 \mathrm{~S}$ gene. However, adjacent to and downstream from this predicted terminus was a 59 bp sequence that was highly conserved between T. rubrum, Trichophyton violaceum, Trichophyton tonsurans and T. mentagrophytes var. interdigitale. The NTS sequences of all four species diverged significantly immediately after this region. The conserved sequence formed part of the 3' external transcribed spacer (ETS), and secondary structure predictions indicated that part of this region could fold into a stable stem-loop structure. A similar structure in Saccharomyces cerevisiae functions in the processing of the pre-rRNA transcript and maturation of the LSU RNA (Allmang \& Tollervey, 1998). An illustration of the conserved region is available in Supplementary Fig. S1 in JMM Online.

\section{Identification of SREs}

Dot-matrix self-comparison of the NTS sequence from isolate 2111 identified three separate regions containing repetitive elements. Each individual repeat unit was identified using co-ordinates from the dot matrix, and also by comparison with known SREs from T. rubrum (Jackson et al., 2000) and T. tonsurans (Gaedigk et al., 2003). The repeats in each of the three regions varied markedly in number, length and sequence homology. The first region, named TmiSO, contained eight copies of a short, highly degenerate repeat that varied in length from 32 to 43 residues, with an interruption of $20 \mathrm{bp}$ in the fourth repeat (Fig. 1). There were no counterparts of the TmiS0 elements in either T. rubrum or T. tonsurans.

The second region, TmiS1, contained two much larger 300 bp repeats, which were homologous to the interrupted variable internal repeats (VIRs) identified in T. tonsurans (Gaedigk et al., 2003). The TmiS1 elements were also located in a very similar region of the NTS to the large TrS1 repeats of T. rubrum (Jackson et al., 2000), but were substantially longer (300 versus $200 \mathrm{bp}$ ) and bore limited sequence 


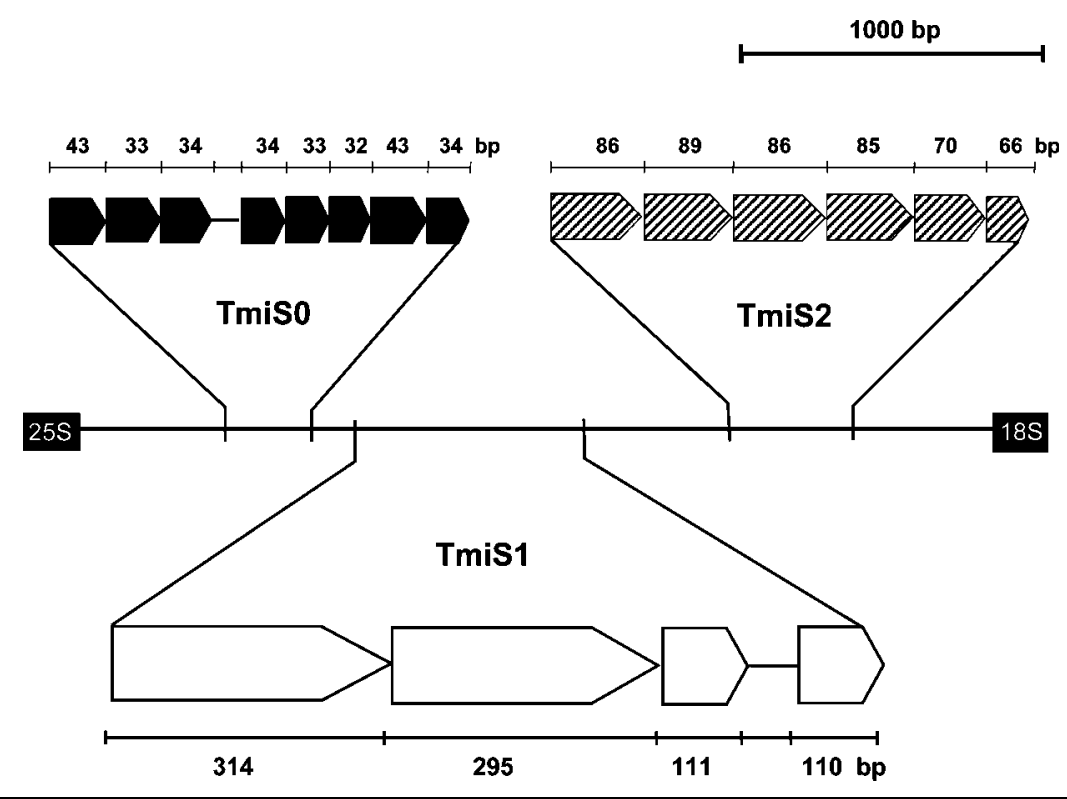

Fig. 1. Diagramatic representation of the NTS region of $T$. mentagrophytes var. interdigitale isolate 2111. The approximate positions of and exact repeat size and copy number for the TmiSo, TmiS1 and TmiS2 SRE loci are indicated. homology to them. Partial or truncated copies of TrS1 exist in the NTS of T. rubrum, and two similarly attenuated copies of the TmiS1 SREs were identified adjacent to and downstream of the main cluster of TmiS1 elements (Fig. 1). Homologues of these partial elements have been categorized as discrete repetitive units in $T$. tonsurans (Gaedigk et al., 2003), but sequence alignments suggest that in T. mentagrophytes var. interdigitale, they are specifically truncated forms of entire TmiS1 repeats.

A limited number of VIRs from T. tonsurans have been found to contain an 'interruption' sequence (Gaedigk et al., 2003). When multiple TmiS1 elements were sequenced from different strains of T. mentagrophytes var. interdigitale, this 'interruption' was present in every copy $(n=12)$, and thus formed an integral part of the TmiS1 repeat structure in this species.

The third region, named TmiS2, contained four full-length repeats of between 85 and 89 bp, and two partial copies of 70 and $66 \mathrm{bp}$, respectively. The TmiS2 repeats showed substantial positional and primary sequence conservation with the TrS2 repeat region of T. rubrum and TvS2 of $T$. violaceum, in contrast to $T$. tonsurans, which contains no repetitive elements at this location. A comparison of the S2 repeats in these species is available in Supplementary Fig. S2 in JMM Online.

\section{Strain identification by PCR fingerprinting of the three Tmi subrepeat loci}

Amplification products from each of the three loci showed different types of strain-specific polymorphisms (Fig. 2a-c). The least polymorphic locus was TmiS2, for which 37/42 isolates $(88 \%)$ had a single-banded PCR product of $684 \mathrm{bp}$. This was designated pattern type II. Two isolates (EP0843 and EK1471) produced a smaller fragment of approximately 600 bp (type I), which was also amplified from the teleomophic species Arthroderma vanbreuseghemii. Two further isolates (EF2608 and EP1174) produced progressively larger fragments of approximately 920 and $1000 \mathrm{bp}$, respectively, designated types III and IV. The TmiS2 pattern types I-IV are illustrated in Fig. 2(c). The size of the TmiS2 elements was approximately $85 \mathrm{bp}$, and hence the type I pattern may represent five repeats; type II, six repeats; type III, ten repeats and type VI, 11 repeats.

The TmiS0 locus showed a higher degree of variability (Fig. 2a), with ten different PCR types identified from the 42 isolates. However, a majority of strains (27/42; 64 \%) again showed one common pattern type (type D), represented by a single 800 bp PCR fragment. The nine other variable types, designated $\mathrm{A}-\mathrm{C}$ and $\mathrm{E}-\mathrm{J}$, were identified either from low numbers of isolates or in single strains only (Table 1). The first six types (A-F) had a single band of variable size, but four isolates (types G-J) had complex patterns containing multiple bands (Fig. 2a). Two isolates from India (SM8774 and SM8779) failed to amplify with these primers. A characteristic low-molecular-mass band of approximately $450 \mathrm{bp}$ was amplified from the teleomorph $A$. vanbreuseghemii (lane B, Fig. 2a). Preliminary sequence data for some of the PCR fragments from types A-E indicate that a combination of repeat element multiplications and insertion/deletion events is responsible for the polymorphisms observed at this locus.

The TmiS1 region showed the greatest variation, but as with the other loci, a single PCR pattern predominated. This pattern, designated type 2, was represented by a 1175 bp PCR fragment containing two copies of the TmiS1 SRE, and was present in 23/42 isolates (55\%). Five simple pattern types (types 1-5) were observed with incremental size variations corresponding to full copies of the TmiS1 element. Five multiple-banded, complex PCR profiles (types 6-10) were identified for eleven isolates (lanes 


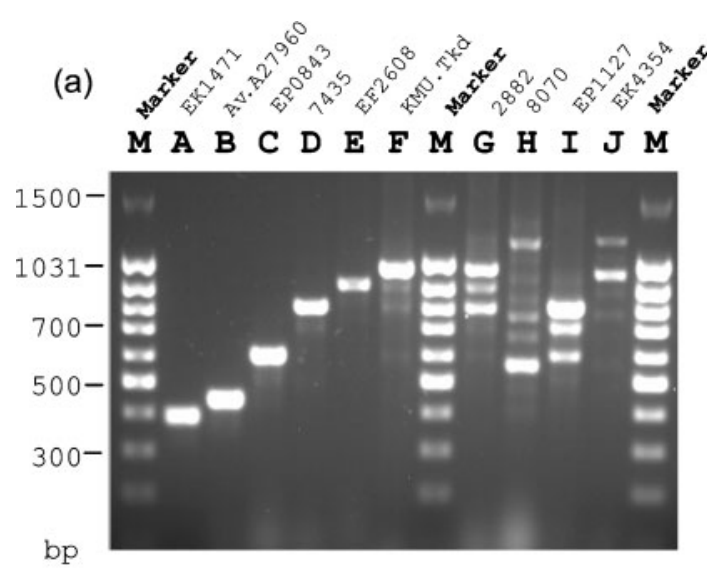

(b)

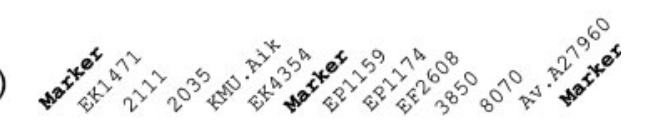

$\begin{array}{llllllllllllll}M & 1 & 2 & 3 & 4 & 5 & \text { M } & 6 & 7 & 8 & 9 & 10 & 0 & \text { M }\end{array}$

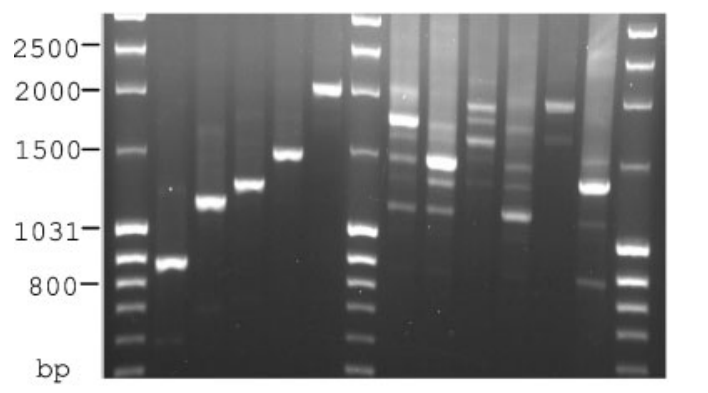

(c)
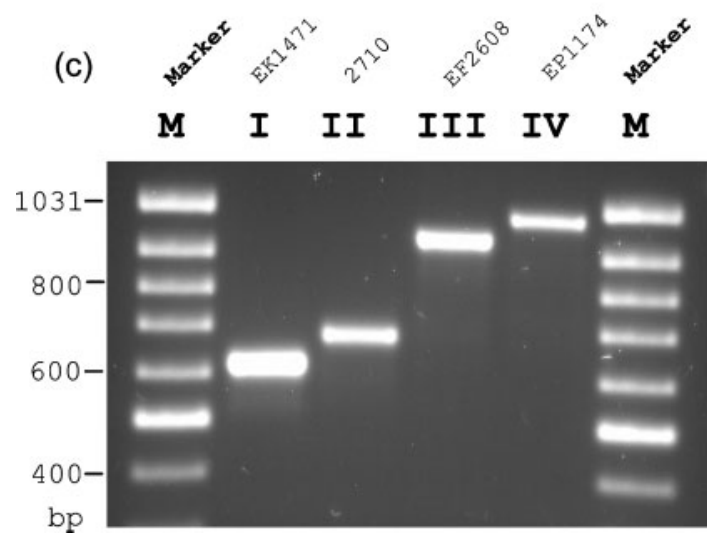

Fig. 2. $P C R$ fingerprint patterns determined for 42 isolates of T. mentagrophytes var. interdigitale at the TmiSo (a), TmiS1 (b) and TmiS2 (c) subrepeat loci.

6-10, Fig. 2b). Two isolates from India (SM8774 and SM8779) again failed to amplify with these primers. A unique, complex pattern (type 0 ; Fig. $2 \mathrm{~b}$, lane 0 ) was amplified from the TmiS1 locus of $A$. vanbreuseghemii.

Pattern variability at each individual repeat locus appeared to occur independently of the other two. By combining the PCR profiles, a total of 19 PCR types were recognized from the 42 isolates (Table 3), with a further unique type for $A$. vanbreuseghemii (pattern 20, type B 0 1; Table 3).
Table 3. Combined So, S1 and S2 fingerprint profiles for 19 PCR types of $T$. mentagrophytes var. interdigitale

Forty-two isolates were assayed, two of which failed to amplify with the S0 and S1 primers. The profile of one isolate of A. vanbreuseghemii is included in the results (marked with an asterisk).

\begin{tabular}{|lcc|}
\hline Number & PCR type & No. isolates (percentage of total) \\
\hline 1 & D 2 II & $16(39)$ \\
2 & D 7 II & $3(7)$ \\
4 & C 2 II & $3(7)$ \\
3 & D 6 II & $2(5)$ \\
5 & F 2 II & $2(5)$ \\
6 & F 1 II & $1(2 \cdot 4)$ \\
7 & D 3 II & $1(2 \cdot 4)$ \\
8 & D 4 II & $1(2 \cdot 4)$ \\
9 & D 6 II & $1(2 \cdot 4)$ \\
10 & D 9 II & $1(2 \cdot 4)$ \\
11 & D 7 IV & $1(2 \cdot 4)$ \\
12 & G 2 II & $1(2 \cdot 4)$ \\
13 & G 1 II & $1(2 \cdot 4)$ \\
14 & E 8 III & $1(2 \cdot 4)$ \\
15 & H 10 II & $1(2 \cdot 4)$ \\
16 & I 7 IA & $1(2 \cdot 4)$ \\
17 & J 5 II & $1(2 \cdot 4)$ \\
18 & C 2 I & $1(2 \cdot 4)$ \\
19 & A 1 I & $1(2 \cdot 4)$ \\
$20^{*}$ & B 0 1 & $1(2 \cdot 4)$ \\
Total & - & $41(100)$ \\
& & \\
\hline
\end{tabular}

Reproducibility was high, with DNA extracted from the same strain by different investigators producing identical PCR profiles. Two isolates from the Indian subcontinent produced fragments with the TmiS2 primers, but could not be typed from the S0 and S1 regions. Phenotypically, these isolates were $T$. mentagrophytes var. interdigitale, and showed ITS restriction profiles characteristic of this species. However, isolates previously identified as T. mentagrophytes var. mentagrophytes (Mochizuki et al., 2003) which were from non-foot sites with patients reporting animal contact as a likely causal factor, also produced an S2 product alone when amplified with the three sets of fingerprinting primers (data not shown). We are therefore further investigating the molecular identity of the Indian strains by sequence analysis of the ITS and NTS regions.

A. vanbreuseghemii is the teleomorphic species from which the sexually degenerate anamorph $T$. mentagrophytes var. interdigitale has evolved (Gräser et al., 1999a). The teleomorph contains low copy numbers of both the TmiS0 and TmiS2 repeats (four and five, respectively) relative to T. mentagrophytes var. interdigitale, and the high repeat copy numbers in the latter therefore appear to have arisen during the course of its host adaptation and divergent evolution from A. vanbreuseghemii. Interestingly, although the closely related species $T$. tonsurans has counterparts of the TmiS1 repeats, it entirely lacks any SREs at the TmiS0 
and TmiS2 positions in the NTS (Gaedigk et al., 2003). Although the TmiS1 SREs are very similar to the VIRs of $T$. tonsurans, the large repeats (the TrS1 elements) that exist at a comparable location in the NTS of T. rubrum bear limited sequence homology to either the TmiS1 or VIR elements. This particular region of the NTS may represent a 'repetition hotspot', as similar types of unrelated repeats appear to have arisen independently in different dermatophyte species. Palindromic sequences are a feature of all these large repeats, and may be involved in a saltatory mechanism of repeat generation. Multiple copies of the TmiS1 elements show high sequence conservation within and between isolates, and appear to be generated by duplicative processes involving the second and subsequent $\mathrm{S} 1$ elements, but not the first or last.

In contrast to the divergence of the TmiS1 and TrS1 repeats, the TrS2 elements in $T$. rubrum have close sequence homology to the corresponding TmiS2 repeats in $T$. mentagrophytes var. interdigitale, and these conserved repetitive regions, proximal to the SSU gene, may have a role in the regulation of rDNA transcription.

\section{Pattern distributions among clinical sites/countries}

There are remarkable parallels between the distribution of PCR types in T. mentagrophytes var. interdigitale and of those in T. tonsurans and T. rubrum. In all three species, a majority of isolates belong to only one PCR type, and this type is characterized by having either one or two copies of the main repeat element. In T. rubrum and T. mentagrophytes var. interdigitale, the repeat locus closest to the SSU rRNA gene (TmiS2, TrS2) shows much less interstrain polymorphism than the second, larger locus (TmiS1, TrS1). The predominance of a single PCR type, and its occurrence in different geographic locations, imply clonality in all these species. In the current study, no clear associations existed between the PCR type and the geographic origin or clinical site of isolates, but a much larger and better-documented sample base is required in order to identify any such links.

\section{Conclusion}

Molecular studies have demonstrated that, as with $T$. rubrum (Gräser et al., 1999b), there is a lack of differentiation between strains of T. mentagrophytes var. interdigitale at the genetic level (Mochizuki et al., 1996). Adapted strains may have spread throughout the human population very rapidly (Charif \& Elewski, 1997), and this recent emergence or 'epidemic' spread of a limited number of strain types has generated the pattern distributions we observe here. It is possible that this explosive increase in dermatophyte population size has influenced the accumulation of polymorphisms in specific genetic markers, such as the rRNA subrepeat elements reported here, since the copy number of such tandemly repetitive regions is regulated by events occurring at mitosis, such as unequal crossover (Szostak \& Wu, 1980). These markers, and other mini- and microsatellites, currently represent the most effective, and often the only practical means, for the molecular subtyping of anthropophilic dermatophytes (Jackson et al., 1999, 2000; Gaedigk et al., 2003; Mochizuki et al., 2003; Ohst et al., 2004). Our report of a rapid, simple and discriminatory method for subtyping T. mentagrophytes var. interdigitale will provide a valuable resource for future investigations into the epidemiology and pathogenesis of this species.

\section{ACKNOWLEDGEMENTS}

This study was supported by a grant from the College Fund of the University of Wales Aberystwyth.

\section{REFERENCES}

Allmang, C. \& Tollervey, D. (1998). The role of the $3^{\prime}$ external transcribed spacer in yeast pre-rRNA processing. J Mol Biol 278, 67-78.

Charif, M. A. \& Elewski, B. (1997). A historical perspective on onychomycosis. Dermatol Ther 3, 43-45.

Drake, L. A., Dinehart, S. M., Farmer, E. R. \& 15 other authors (1996). Guidelines of care for superficial mycotic infections of the skin: tinea corporis, tinea cruris, tinea faciei, tinea manuum, and tinea pedis. J Am Acad Dermatol 34, 282-286.

Gaedigk, A., Gaedigk, R. \& Abdel-Rahman, S. M. (2003). Genetic heterogeneity in the rRNA gene locus of Trichophyton tonsurans. J Clin Microbiol 41, 5478-5487.

Gräser, Y., Kuijpers, A. F. A., Presber, W. \& De Hoog, G. S. (1999a). Molecular taxonomy of Trichophyton mentagrophytes and $T$. tonsurans. Med Mycol 37, 315-330.

Gräser, Y., Kuhnisch, J. \& Presber, W. (1999b). Molecular markers reveal exclusively clonal reproduction in Trichophyton rubrum. J Clin Microbiol 37, 3713-3717.

Jackson, C. J., Barton, R. C. \& Evans, E. G. V. (1999). Species identification and strain differentiation of dermatophyte fungi by analysis of ribosomal DNA intergenic spacer regions. J Clin Microbiol 37, 931-936.

Jackson, C. J., Barton, R. C., Kelly, S. L. \& Evans, E. G. V. (2000). Strain identification of Trichophyton rubrum by specific amplification of subrepeat elements in the ribosomal DNA nontranscribed spacer. J Clin Microbiol 38, 4527-4534.

Mochizuki, T., Watanabe, S. \& Uehara, M. (1996). Genetic homogeneity of Trichophyton mentagrophytes var. interdigitale isolated from geographically distant regions. J Med Vet Mycol 34, 139-143.

Mochizuki, T., Ishizaki, H., Barton, R. C., Moore, M. K., Jackson, C. J., Kelly, S. L. \& Evans, E. G. (2003). Restriction fragment length polymorphism analysis of ribosomal DNA intergenic regions is useful for differentiating strains of Trichophyton mentagrophytes. J Clin Microbiol 41, 4583-4588.

Ohst, T., de Hoog, S., Presber, W., Stavrakieva, V. \& Gräser, Y. (2004). Origins of microsatellite diversity in the Trichophyton rubrum-T. violaceum clade (dermatophytes). J Clin Microbiol 42, 4444-4448.

Smith, E. S., Fleischer, A. B. \& Feldman, S. R. (1998). Nondermatologists are more likely than dermatologists to prescribe antifungal/corticosteroid products: an analysis of office visits for cutaneous fungal infections, 1990-1994. J Am Acad Dermatol 39, 43-47. 
Szostak, J. \& Wu, R. (1980). Unequal crossing over in the ribosomal DNA of Saccharomyces cerevisiae. Nature 284, 426-430.

Takiuchi, I., Morishita, N., Hamaguchi, T., Ninomiya, J. \& Higuchi, R. (2005). Treatment outcome and relapse with short-term oral terbinafine $\left(250 \mathrm{mg} \mathrm{day}^{-1}\right)$ in tinea pedis. Nippon Ishinkin Gakkai Zasshi 46, 285-289.

Tosti, A., Piraccini, B. M., Stinchi, C. \& Colombo, M. D. (1998). Relapses of onychomycosis after successful treatment with systemic antifungals: a three-year follow-up. Dermatology 197, 162-166.
White, T. J., Bruns, T. \& Taylor, J. (1990). Amplification and direct sequencing of fungal ribosomal RNA genes for phylogenetics. In PCR Protocols: a Guide to Methods and Applications. Part 3: Genetics and Evolution, Chapter 38, pp. 315-322. Edited by M. A. Innis, D. H. Gelfand, J. J. Sninsky \& T. J. White. London: Academic Press.

Zaias, N. \& Rebell, G. (2003). Clinical and mycological status of the Trichophyton mentagrophytes (interdigitale) syndrome of chronic dermatophytosis of the skin and nails. Int J Dermatol 42, 779-788. 